\title{
Soil Chemistry and Mineralogy of the Santa Cruz Coastal Terraces
}

By Colin Pinney ${ }^{1}$, Jacob Aniku ${ }^{3}$, Raymond Burke ${ }^{2}$, Jennifer Harden ${ }^{1}$, Michael

Singer $^{3}$, and Jennie Munster ${ }^{1}$

${ }^{1}$ U.S. Geological Survey, 345 Middlefield Rd. MS 962, Menlo Park, CA 94025.

${ }^{2}$ California State University, Humboldt.

${ }^{3}$ University of California, Davis.

Table of Contents

1.0 Introduction

2.0 Geologic setting

3.0 Field and laboratory procedures

4.0 Data set descriptions (in parenthesis is the name of the downloadable file)

4.1 Table 1 Locations of sample sites

4.2 Table 2a,b Field descriptions

4.3 Table 3 Physical properties

4.4 Table 4 Extractive chemical analyses

4.5 Table 5 Mineralogy

4.6 Table 6 Total chemical analyses of the fine $(<47 \mu \mathrm{m})$ fraction by $\mathrm{X}$ ray fluorescence

4.7 Table 7 Total chemical analyses of the $<2-\mathrm{mm}$ fraction by X-ray fluorescence

5.0 Reference

6.0 Acknowledgements 


\subsection{Introduction}

Marine terraces in the central coast of California provide an opportunity to study a soil chronosequence in which similar materials (beach deposits) have been weathered under similar slope, climatic, and vegetation conditions during the Quaternary. The terraces between Santa Cruz and An_o Nuevo, California have been studied for decades and are thought to be one of the best example of marine terraces in California \{Lawson (1893), Wilson (1907); Branner and others (1909), Rode (1930) Page and Holmes (1945), Alexander (1953), Bradley (1956, 1957, 1958, and 1965), Bradley and Addicott (1968), Clark (1966 and 1970), Jahns and Hamilton (1971), Lajoie and others (1972), Bradley and Griggs (1976). Hanks and others (1986), Aniku (1986), Fine and others (1988), Anderson (1990 and 1994), and Rosenbloom and Anderson (1994).\} Here we report morphological, chemical, physical and mineralogical data for the soils that were formed in deposits on the Santa Cruz marine terraces in order to examine soil characteristics as a function of increasing terrace age.

\subsection{Geologic setting}

The main characteristics of terrace formation include (1) deposition of sediment while the platform is still submerged (2) platforms, formed by wave energy, are cut into bedrock during sea-level high stands (3) as sea level drops beach sediment, colluvium, and local stream alluvium are deposited onto the newly exposed bedrock platform and (4) terraces are tectonically uplifted, deformed, eroded and incised (Bradley and Griggs 1976). In general, the terraces slope seaward at low angles and with a slight concavity. Although processes that form and deform the terraces are well understood, the time of deposition and stabilization of the terrace surface, and the exact rates of the tectonic uplift, are less well known (Bradley and Griggs 1976, Hanks and others 1986, Perg and others 1999). In general, however, terraces increase in age from the lowest terrace that forms the current ocean blufftop to the highest terrace that occurs $\sim 4 \mathrm{~km}$ inland and $\sim 500$ $\mathrm{m}$ in elevation.

There are five discernable terraces between Santa Cruz and An_o Nuevo, California defined by Bradley and Griggs (1976), in order from oldest to youngest, as Quarry, Blackrock, Wilder, Western and Highway 1. Only the Highway 1 terrace is 
spatially continuous. Regional uplift rates appear to be more rapid toward the south, as a result, identification and correlation of specific terraces is problematic especially where fewer than five terraces are present (Anderson, 1990, 1994). Although we refer to our sites using the nomenclature of Bradley and Griggs, we caution that absolute ages and even the assignment of terrace names are tenuous until more dating information becomes available. Our identification of the top two terraces, Quarry terrace and Black Rock (Table 1) are particularly suspect. The two sites could conceivably be front and back edges of one terrace (Quarry) or indeed are two separate terraces.

The present climate is Mediterranean with cool, wet winters $\left(9\right.$ to $\left.10^{\circ} \mathrm{C}\right)$ and warm dry summers $\left(16\right.$ to $\left.17^{\circ} \mathrm{C}\right)$ with the influence of fog. The climate of the late Pleistocene, when the marine terraces were cut, was similar to present day with only moderately cooler, wetter conditions during glacial periods (Gorsline et al. 1976, Johnson, 1977, Sims et al., 1981). The vegetation at present, on the terraces is composed of annual grasses and oak with redwood trees found in the stream channels.

There are numerous faults traversing these terraces from the North AmericanPacific plate boundary (Anderson, 1990, 1994). Tectonics associated with these faults is one of the major components in forming marine terraces. The uplift rates along the coast according to Bradley and Griggs (1976) vary from 0.16 to $0.48 \mathrm{~m} \mathrm{kyr}^{-1}$, but may be as fast as $1.15 \mathrm{~m} \mathrm{kyr}^{-1}$ (Perg and others, 1999) Understanding rates of uplift aid in dating the terraces and soil profiles.

Underlying the terraces is Tertiary Santa Cruz mudstone and Santa Margarita sandstone. These are easily eroded by waves and streams, but remain intact in interfluves (Clark, 1981: Bradley and Griggs, 1976). The Santa Cruz Mudstone is a thin to mediumbedded, brown and gray to light-gray, buff, and light-yellow siliceous mudstone with nonsiliceous mudstone and siltstone and minor amounts of sandstone. The siliceous nature implies organic deposition in a quiescent, deep-water environment. The Santa Margarita sandstone is a thin, bioturbated light-gray to grayish-orange to white, friable, very fine-to very coarse-grained arkosic sandstone. Fine-grained sandstone commonly contains glauconite. A quartz and feldspar pebble conglomerate crops out locally at the base of the section. The Santa Margarita Sandstone represents a shallow marine deposit (Barnes and others 2000). 


\subsection{Field and laboratory procedures}

Conventional methods were used to describe, sample and analyze soils from the Santa Cruz terraces. Briefly, soil profiles were sampled by genetic horizons from backhoe pits and drill cores. Physical, extractive chemical, and total chemical analyses are reported in appendices following the conventions of Harden and others (1986), USGS Bulletin series 1590. A detailed explanation of our field and analytical methods is included in Singer and Janitzky (1986). Methods for iron and clay mineralogy are described in J.R. Aniku (1986).

\subsection{Data set descriptions}

4.1 Table 1 Locations of Sample Sites List UTM coordinates and elevation for all terrace sample sites.

4.2 Table 2 Field descriptions

Table 2a, Field description abbreviation explanations: This table describes the abbreviations used in the following table. This includes explanations for soil structure, texture, consistence, horizon boundaries, roots and pores, and clay films.

Table 2b, Field description: A "--" indicates that property was not described. This table list the properties described in the field for all soil horizons and consist of: No.-A numeric sequence to quickly cross compare data from table to table Sample-Descriptor of pit Horizon-General description of the sampled horizon according to conventions of the Soil Survey Staff (1998) Depth-Depth of upper and lower boundaries, in $\mathrm{cm}$, of the horizon Lower Boundary-Description of contact with lower boundary according to conventions of the Soil Survey Staff (1998)

Munsell Color-Moist and dry color according to the Munsell color chart. Texture-Soil texture class following conventions of Soil Survey Staff (1998), a + indicates more clay 
Structure-Soil structure strength, size and type according to conventions of Soil Survey Staff (1998)

Consistence-Consistence according to conventions of Soil Survey Staff (1998), described for dry, moist and wet consistence

Roots-Root abundance and size according to conventions of Soil Survey Staff, (1998)

Pores-Pore abundance and size according to conventions of Soil Survey Staff, (1998)

Clay films-Clay frequency, thickness and morphology according to conventions of Soil Survey Staff (1998)

$\mathrm{pH}-$ The $\mathrm{pH}$ of the sample as determined in the field

4.3 Table 3 Physical properties

This table yields data from particle size analysis delineated according to classification of Soil Survey Staff, (1998). The first three columns are the same as the Table 2. The other columns are as follows:

Basal depth-Lower depth of sample horizon in $\mathrm{cm}$

Bulk Density - Grams soil per cubic centimeter on an oven-dry basis

Texture-Texture according to the particle size distribution and as defined by Soil Survey Staff (1998)

4.4 Table 4 Extractive chemical analyses

The first three columns are the same as the Table 2. Analyses performed on selected samples, $<2 \mathrm{~mm}$ fraction, quantifying, organic carbon, cation exchange capacity, $\mathrm{Fe}, \mathrm{Al}, \mathrm{pH}$, exchangeable $\mathrm{Na}, \mathrm{K}, \mathrm{Ca}, \mathrm{Mg}$, and $\mathrm{N}$.

4.5 Table 5 Mineralogy Analyses performed selected samples to determined presence of Kaolinite, Mica, Chlorite-Vermiculite, Gibbsite, expanding clays, Quartz and Feldspar.

4.6 Table 6 Total chemical analyses of the fine $(<47 \mu \mathrm{m})$ fraction by X-ray fluorescence.

The first three columns are the same as the Table 2. Analyzed for Quartz $\left(\mathrm{SiO}_{2}\right)$, Alumium Oxide $\left(\mathrm{Al}_{2} \mathrm{O}_{3}\right)$, Iron Oxide $\left(\mathrm{Fe}_{2} \mathrm{O}_{3}\right)$, Magnesium Oxide $(\mathrm{MgO})$, Calcium Oxide $(\mathrm{CaO})$, Sodium Oxide $\left(\mathrm{Na}_{2} \mathrm{O}\right)$, Potassium Oxide $\left(\mathrm{K}_{2} \mathrm{O}\right)$, Titanium Dioxide 
$\left(\mathrm{TiO}_{2}\right)$, Phosphorus Pentoxide $\left(\mathrm{P}_{2} \mathrm{O}_{5}\right)$, Manganous Oxide $(\mathrm{MnO})$, Zirconium Dioxide $\left(\mathrm{ZrO}_{2}\right)$.

4.7 Table 7 Total chemical analyses of the $<2 \mathrm{~mm}$ fraction by X-ray fluorescence.

The first three columns are the same as the Table 2. Analyzed for Quartz $\left(\mathrm{SiO}_{2}\right)$, Alumium Oxide $\left(\mathrm{Al}_{2} \mathrm{O}_{3}\right)$, Iron Oxide $\left(\mathrm{Fe}_{2} \mathrm{O}_{3}\right)$, Magnesium Oxide $(\mathrm{MgO})$, Calcium Oxide $(\mathrm{CaO})$, Sodium Oxide $\left(\mathrm{Na}_{2} \mathrm{O}\right)$, Potassium Oxide $\left(\mathrm{K}_{2} \mathrm{O}\right)$, Titanium Dioxide $\left(\mathrm{TiO}_{2}\right)$, Phosphorus Pentoxide $\left(\mathrm{P}_{2} \mathrm{O}_{5}\right)$, Manganous Oxide $(\mathrm{MnO})$, Zirconium Dioxide $\left(\mathrm{ZrO}_{2}\right)$.

\subsection{References}

Alexander,C.S., 1953, The marine and stream terraces of the Capitola-Watsonville area: California University Publications Geography, v. 10, p. 1-44.

Anderson, R.S., 1990, Evolution of the northern Santa Cruz Mountains by advection of crust past a San Andreas Fault bend.: Science, v. 249, p. 397-401. 1994, Evolution of the Santa Cruz Mountains, California, through tectonic growth and geomophic decay.: Journal of Geophysical Research, v. 99, no. B10, p. 20161-20179.

Aniku, Jacob Robert Francis, 1986, Trends of pedogenic iron oxides in a marine terrace chronosequence. Davis, University of California, Ph.D. dissertation, 227 p.

Barnes, J. Roberts, S., and Mills, S., 2000, Description of map units for the geology of the Pescadero/Butano creek watershed, and the Half Moon Bay and Montara Mountain quadrangles, San Mateo County, California. USGS Open-File Report 00-127..

Bradley, W.C., 1956, Carbon-14 date for a marine terrace at Santa Cruz, California: Geological Society of America Bulletin, v. 67, p. 675-677 1957, Origin of marine-terrace deposits in the Santa Cruz area, California: Geological Society of America Bulletin, v.67, p. 421-444. 1958, Submarine abrasion and wave-cut platforms: California: Geological Society of America Bulletin, v.69, p. 967-974. 
1965, Marine Terraces on Ben Lomond Mountains, California, in Wahrhaftig, C., and others, eds., Internat. Assoc. Quaternary Research $7^{\text {th }}$ Cong., field conf. I guidebook, Northern Great Basin and California: p148-150.

Bradley, W.C. and Addicott, W.O. 1968, Age of first marine terrace near Santa Cruz, California: California: Geological Society of America Bulletin, v. 79, p. 12031210.

Bradley, W.C. and Griggs, G.B. 1976, Form, genesis, and deformation of central California wave-cut platforms: California: Geological Society of America Bulletin, v. 87, p. 433-449.

Branner, J.C., Newsom, J.F., and Arnold, Ralph, 1909, Santa Cruz folio: U.S. Geol. Survey and Geol. Atlas, Folio 163, 11 p.

Bryant, R.B., N. Curi, C. B. Roth, and Franzmeir, D.P., 1983. Use of internal standard with differential X-ray diffraction analysis for iron oxides. Soil Sci Soc. Amer. J. 47: $168-173$.

Clark, J.C., 1966, Terriary stratigraphy of the Felton-Santa Cruz area, Santa Cruz Mountains, California: Stanford, Calif., Stanford University, Ph.D. dissertation, $184 \mathrm{p}$.

Fine, P., Singer,M.J. 1989, Contribution of ferrimagnetic minerals to oxalate extractable and dithionite extractable iron.: Soil Science Society of America Journal, v. 53, no. 1, p. 191-196

Gorsline, D.S., Pao, G.A., Prensky, S. E., and Mulhern, M., 1976, High resolution studies of paleo-climatic and paleo-oceanographic history in California Contental Borderland basin sediments, AAPG Bull., 60, 675.

Hanks, T.C., Bucknam, R.C., Lajoie, K.R., Wallace, R.E., 1984, Modification of WaveCut and Faulting-Controlled Landforms: Journal of Geophysical Research, v. 89, no. B7, p. 5771-5790.

Harden, J.W., 1986, Soils developed in granitic alluvium near Merced, California, in Harden, J. W., (ed): A series of soil chronosequences in the western United States U.S. Geological Survey Bulletin 1590A, 35p

Johnson, D.L., The late Quaternary climate of coastal California: Evidence for an ice age refugium, Quat. Res., 8, 154-179, 1977 
Lajoie, K.R., Weber, G.E., and Tinsley, J.C., 1972, Marine terraces deformation: San Mateo and Santa Cruz Counties, in Progress report on USGS Quaternary studies in San Francisco Bay area, an informal collection of preliminary papers, Friends of Pleistocene guidebook: p.100-113.

Lawson, A.C., 1893, The post-Pliocene diastrophism of the coast of southern California: California University Department of Geological Science Bulletin, v1, p.115- 160.

Page, B.M. and Holmes, C.N., 1945, Geology of the bituminous sandstone deposits near Santa Cruz, Santa Cruz County, California: U.S. Geological Survey Oil and Gas Inv. Prelim. Map 27.

Perg, L.A., Anderson, R.S., and Finkel, R.C., 1999, 10Be and 26Al dating of the Santa Cruz Marine Terraces, California.: EOS transactions, AGU Supplement, v. 80, no. 46, p. F443.

Rode, Karl, 1930, Geomophogenie des Ben Lomond (Kaliforien), eine Studie über Terrassenbildung durch marine Abrasion: Zeitschr. Geomorphologie, v. 5, p.1678.

Rosenbloom, N.A. and Anderson, R.S. 1994, Hillslope and channel evolution in a marine terraced landscape, Santa Cruz, California.: Journal of Geophysical Research, v. 99, no B7, p. 14013-14029.

Schulze, D.G. 1981. Identification of iron oxide minerals by differential X-ray diffaction. Soil Sci. Soc. Am. J. 45: 437-440.

Sims, J.D. Adam, D.P., and Rymer, M.J., Late Pleistocene stratigraphy and palynology of Clear Lake, in Research in Ceysers-Clear Lake Geothermal Area, Northern California, U.S. Geological Survey Prof. Pap. 1141, edited by R.J. McLaughlin and J.M. Donnelly-Nolan, 219-230, 1981.

Singer, M.J and Janitzky, P, Editors Field and Laboratory Procedures Used in a Soil Chronosequence Study, U.S. Geological Survey Bulletin 1648.

Soil Survey Staff, 1998. Keys to Soil Taxonomy. U.S. Dept. Agric., $8^{\text {th }}$ ed, 326 p. Washington D.C.

Wilson, M.E., 1907, Shore topography near Davenport, Santa Cruz County: California Physical Geography Club Bulletin, v. 1, p. 1-14. 


\subsection{Acknowledgements}

In memory of Denis Marchand, who died suddenly just months before this field excursion. Denny was leader and mentor for the USGS project on soil chronosequences of the Western U.S. We thank our field party Mike Machette, Marith Reheis, Alan Busacca, Emily Taylor, Bill Bradley, Sam Schaler, and Bob Curry for their generous help and support. We also thank Peter Janitzky and USGS analysts (A.J. Bartel, K. Stewart, J. Taggart, R. Johnson, K. Dennen under J.R. Lindsay) for their standard of excellence in laboratory procedures. 
Table 1

Table 1. Locations of Sample Sites Terraces names based on Bradley and Griggs (1976)

\begin{tabular}{|c|c|c|}
\hline Sample Site & UTM Coordinates & Elevation \\
\hline $\begin{array}{l}\text { Highway 1 (Davenport) Terrace } \\
\text { Site SC2 }\end{array}$ & $\begin{array}{l}10 \mathrm{~s} 0586434 \mathrm{e} \\
4090149 \mathrm{n}\end{array}$ & $14 \mathrm{~m}$ \\
\hline Western Terrace & Not Sampled & $\begin{array}{l}\text { Not } \\
\text { Sampled }\end{array}$ \\
\hline $\begin{array}{l}\text { Wilder Terrace } \\
\text { Site SC3 (upper site) } \\
\text { Site SC4 (lower site) } \\
\text { Site SC5 (lower site) }\end{array}$ & $\begin{array}{l}\text { 10s 0584467e } \\
4093212 \mathrm{n} \\
10 \mathrm{~s} 0584486 \mathrm{e} \\
4092971 \mathrm{n} \\
10 \mathrm{~s} 0584486 \mathrm{e} \\
4092971 \mathrm{n}\end{array}$ & $\begin{array}{l}126 \mathrm{~m} \\
116 \mathrm{~m} \\
116 \mathrm{~m}\end{array}$ \\
\hline $\begin{array}{l}\text { Black Rock Terrace } \\
\text { Site SC1 } \\
\text { Site SC7 } \\
\text { Site SC9 }\end{array}$ & $\begin{array}{l}10 \mathrm{~s} 0583143 \mathrm{e} \\
4093268 \mathrm{n} \\
10 \mathrm{~s} 0583143 \mathrm{e} \\
4093268 \mathrm{n}\end{array}$ & $\begin{array}{l}138 \mathrm{~m} \\
138 \mathrm{~m}\end{array}$ \\
\hline $\begin{array}{l}\text { Quarry/ Black Rock Terrace } \\
\text { Site SC } 6 \\
\text { Site SC8 }\end{array}$ & $\begin{array}{l}\text { 10s } 0582905 \mathrm{e} \\
4093610 \mathrm{n}\end{array}$ & $159 \mathrm{~m}$ \\
\hline
\end{tabular}


Field description abbreviation explanations

Grade
m; massive
sg; single grained
1; week
2; moderate
3; strong

SOIL STRUCTURE

Size

vf; very fine ( $v$ thin)

$f$; fine (thin)

m; medium

c; course (thick)

$\mathrm{vc}$; very coarse ( $\mathrm{v}$ thick)

*If two structures-listed as primary and secondary

\section{SOIL TEXTURE}

\section{$\underline{\text { Size }}$}

co; coarse

f; fine

vf; very fine

Texture

S; sand

LS; loamy sand

SL; sandy loam
L; Loam

SiL; silt loam

Si; silt

\section{SOIL CONSISTENCE}

\section{Dry}

lo; loose

so; soft

sh; slightly hard

$\mathrm{h}$; hard

vh; very hard

eh; extremely hard

\section{Distinctness}

va; very abrupt

a; abrupt

c; clear

g; gradual

d; diffuse

\section{Size}

vf; very

$f ;$ fine

m; medium

co; coarse

Frequency
v1; very few
1; few
2; common
3; many
4; continuous

vf; very firm

efi; extremely firm

HORIZON BOUNDARIES

Topography

s; smooth

w; wavy

i; irregular

b; broken

ROOTS AND PORES

Abundance

1; few

2; common

3; many

CLAY FILMS

Thickness

$\mathrm{n}$; thin

mk; moderately thick

$\mathrm{k}$; thick
Type

gr; granular

pl; platey

pr; prismatic

cpr; columnar

abk; angular blocky

sbk; subangular blocky

\section{Texture}

SCL; sandy-clay loam

$\mathrm{CL}$; clay loam

SiCL; silty-clay loam

SC; sandy clay

C; clay

$\mathrm{SiC}$; silty clay

\section{Wet}

so; nonsticky

ss; slightly sticky

s; sticky

vs; very sticky
Wet

po; nonplastic ps; slightly plastic $p$; plastic vp; very plastic

\section{Pore Shape}

tub; tubular

ir; irregular

v; vesicular

\section{Morphology}

of; ped face coatings

br; bridging grains

po; pore linings

(w; occours as waves or lamellae)

co; coats on clasts 
Table 3. Physical properties

\begin{tabular}{|c|c|c|c|c|c|c|c|c|c|c|c|c|c|}
\hline No. & Sample & Horizon & $\begin{array}{c}\text { Basal } \\
\text { Depth } \\
(\mathrm{cm})\end{array}$ & $\begin{array}{c}\text { Bulk } \\
\text { Density } \\
\left(\mathrm{g} / \mathrm{cm}^{3}\right)\end{array}$ & Texture & $\begin{array}{c}\text { Total } \\
\text { sand } \\
(\%)\end{array}$ & $\begin{array}{c}\text { Very } \\
\text { coarse } \\
\text { sand } \\
(\%)\end{array}$ & $\begin{array}{c}\text { Coarse } \\
\text { sand } \\
(\%)\end{array}$ & $\begin{array}{c}\text { Medium } \\
\text { sand } \\
(\%)\end{array}$ & $\begin{array}{c}\text { Fine, very } \\
\text { fine sand } \\
(\%)\end{array}$ & $\begin{array}{l}\text { Silt } \\
(\%)\end{array}$ & $\begin{array}{c}<2-\mathrm{mm} \\
\text { clay } \\
(\%)\end{array}$ & $\begin{array}{c}<1-\mathrm{mm} \\
\text { clay } \\
(\%)\end{array}$ \\
\hline \multicolumn{14}{|c|}{ Alluvium of Davenport terrace } \\
\hline 1 & $\mathrm{SC} 2$ & A1 & 29 & 1.57 & sandy loam & 69.03 & 0.94 & 3.77 & 8.80 & 55.52 & 21.71 & 9.26 & 7.78 \\
\hline 2 & SC2 & A2 & 50 & 1.70 & sandy loam & 70.08 & 0.10 & 2.48 & 6.83 & 60.66 & 20.26 & 9.66 & 4.46 \\
\hline 3 & SC2 & B1 & 90 & 1.76 & sandy clay loam & 68.33 & 0.62 & 3.85 & 8.84 & 55.02 & 17.05 & 14.62 & 13.56 \\
\hline 4 & SC2 & B2 & 106 & 1.96 & sandy clay loam & 66.80 & 0.10 & 2.47 & 7.73 & 56.49 & 16.30 & 16.90 & 13.93 \\
\hline 5 & SC2 & B3 & 170 & 1.65 & sandy clay loam & 83.67 & 0.00 & 1.12 & 12.30 & 70.25 & 3.02 & 13.31 & 12.24 \\
\hline 6 & SC2 & C10x & 200 & 1.66 & sand & 96.36 & 0.00 & 3.13 & 20.61 & 72.62 & 1.74 & 1.90 & 1.68 \\
\hline 7 & SC2 & IIC2 & 457 & -- & sand & 97.47 & 0.20 & 0.91 & 5.99 & 90.36 & 1.60 & 0.93 & 0.89 \\
\hline 8 & SC2 & $\mathrm{IICn}$ & 518 & -- & sand & 97.47 & 0.00 & 1.32 & 5.36 & 90.79 & 1.24 & 1.29 & 1.18 \\
\hline \multicolumn{14}{|c|}{ Alluvium of Wilder terrace } \\
\hline 9 & SC3 & A11 & 8 & 1.32 & clay loam & 45.10 & 1.13 & 8.76 & 11.68 & 23.54 & 32.90 & 22.00 & 15.20 \\
\hline 10 & SC3 & A12 & 23 & 1.51 & clay loam & 43.92 & 2.04 & 9.56 & 10.96 & 21.36 & 33.94 & 22.14 & 15.19 \\
\hline 11 & SC3 & A3 & 87 & 1.50 & clay loam & 41.03 & 1.49 & 7.93 & 10.60 & 21.01 & 35.26 & 23.71 & 15.94 \\
\hline 12 & SC3 & IIB21t & 126 & 1.57 & clay loam & 41.75 & 1.99 & 8.85 & 10.64 & 20.28 & 31.53 & 26.72 & 20.83 \\
\hline 13 & SC3 & IIB22t & 190 & 1.82 & clay loam & 52.09 & 3.28 & 11.43 & 13.92 & 23.46 & 23.93 & 23.98 & 19.56 \\
\hline 14 & SC3 & IIIB23t & 240 & 1.77 & clay & 51.94 & 2.87 & 14.91 & 15.46 & 18.70 & 21.06 & 27.00 & 22.04 \\
\hline 15 & SC3 & IIIB31 & 325 & 1.90 & sandy clay & 55.68 & 1.84 & 15.15 & 18.12 & 20.57 & 19.45 & 24.87 & 19.81 \\
\hline 16 & SC3 & IIIB32 & 390 & 1.88 & sandy clay & 65.10 & 1.22 & 15.82 & 21.63 & 26.43 & 10.58 & 24.32 & 20.12 \\
\hline 28 & SC5 & All & 2.5 & -- & sandy clay loam & 57.99 & 0.11 & 10.79 & 17.67 & 29.42 & 24.71 & 17.30 & 13.63 \\
\hline 29 & SC5 & A12 & 25 & 1.69 & sandy clay loam & 53.26 & 1.05 & 11.45 & 15.02 & 25.74 & 24.98 & 21.76 & 16.85 \\
\hline 30 & SC5 & B1 & 60 & 1.56 & clay & 48.96 & 1.03 & 11.57 & 14.05 & 22.31 & 21.54 & 29.50 & 25.25 \\
\hline 31 & SC5 & B21t & 85 & 1.66 & clay & 48.46 & 1.29 & 12.59 & 15.46 & 19.13 & 12.49 & 39.05 & 35.00 \\
\hline 32 & SC5 & B22t & 111 & 1.75 & clay & 41.32 & 1.16 & 9.99 & 13.46 & 16.72 & 10.48 & 48.20 & 45.26 \\
\hline 33 & SC5 & B31t & 158 & 1.66 & clay & 52.79 & 1.14 & 12.19 & 17.15 & 22.31 & 14.90 & 32.31 & 30.83 \\
\hline 34 & SC5 & B32 & 220 & 1.60 & clay & 57.32 & 1.26 & 10.33 & 18.23 & 27.50 & 13.26 & 29.42 & 27.95 \\
\hline 35 & SC5 & B33 & 270 & 1.67 & sandy clay & 58.64 & 0.86 & 9.18 & 19.01 & 29.59 & 12.63 & 28.73 & 25.96 \\
\hline 36 & SC5 & B34 & 325 & 1.65 & sandy clay & 59.31 & 0.43 & 5.30 & 15.48 & 38.10 & 11.38 & 29.31 & 26.71 \\
\hline 37 & SC5 & B35 & 415 & 1.51 & sandy clay loam & 65.38 & 0.48 & 4.22 & 9.65 & 51.02 & 13.29 & 21.33 & 18.96 \\
\hline 38 & SC5 & Cox & 480 & 1.51 & sandy clay loam & 72.08 & 4.65 & 17.58 & 20.58 & 29.27 & 9.02 & 18.90 & 16.84 \\
\hline 39 & SC5 core & Cox & 640 & -- & loamy sand & 82.56 & 0.10 & 0.71 & 3.65 & 78.10 & 11.68 & 5.76 & 3.08 \\
\hline
\end{tabular}




\begin{tabular}{|c|c|c|c|c|c|c|c|c|c|c|c|c|c|}
\hline No. & Sample & Horizon & $\begin{array}{c}\text { Basal } \\
\text { Depth } \\
(\mathrm{cm})\end{array}$ & $\begin{array}{c}\text { Bulk } \\
\text { Density } \\
\left(\mathrm{g} / \mathrm{cm}^{3}\right)\end{array}$ & Texture & $\begin{array}{c}\text { Total } \\
\text { sand } \\
(\%)\end{array}$ & $\begin{array}{c}\text { Very } \\
\text { coarse } \\
\text { sand } \\
(\%)\end{array}$ & $\begin{array}{c}\text { Coarse } \\
\text { sand } \\
(\%)\end{array}$ & $\begin{array}{c}\text { Medium } \\
\text { sand } \\
(\%)\end{array}$ & $\begin{array}{c}\text { Fine, very } \\
\text { fine sand } \\
(\%)\end{array}$ & $\begin{array}{l}\text { Silt } \\
(\%)\end{array}$ & $\begin{array}{c}<2-\mathrm{mm} \\
\text { clay } \\
(\%)\end{array}$ & $\begin{array}{c}<1-\mathrm{mm} \\
\text { clay } \\
(\%)\end{array}$ \\
\hline 40 & SC5 core & Cox & 762 & -- & loamy sand & 84.64 & 0.63 & 1.04 & 3.24 & 79.73 & 9.09 & 6.27 & 3.89 \\
\hline 41 & SC5 core & Cox & 823 & -- & loamy sand & 85.14 & 0.21 & 8.88 & 3.41 & 72.65 & 10.28 & 4.58 & 2.02 \\
\hline 42 & SC5 core & Cox & 1128 & -- & sandy loam & 65.40 & 1.65 & 3.40 & 2.06 & 58.29 & 23.52 & 11.08 & 6.88 \\
\hline \multicolumn{14}{|c|}{ Alluvium of Black Rock terrace } \\
\hline 43 & SC1 & A11 & 3 & -- & sandy clay loam & 56.00 & 2.04 & 10.41 & 19.23 & 24.32 & 26.35 & 17.65 & 14.71 \\
\hline 44 & SC1 & A12 & 50 & 1.52 & clay loam & 52.19 & 1.55 & 9.59 & 17.33 & 23.72 & 27.61 & 20.20 & 17.87 \\
\hline 45 & SC1 & B11 & 65 & 1.67 & sandy clay loam & 56.23 & 1.99 & 10.74 & 18.37 & 25.13 & 22.37 & 21.63 & 20.68 \\
\hline 46 & SC1 & B12 & 84 & 1.65 & clay loam & 52.26 & 1.21 & 9.36 & 19.13 & 22.56 & 22.60 & 25.14 & 23.00 \\
\hline 47 & SC1 & B21t & 128 & 1.70 & clay & 49.43 & 1.15 & 10.35 & 20.59 & 17.35 & 11.27 & 39.30 & 36.61 \\
\hline 48 & SC1 & B22t & 195 & 1.68 & sandy clay & 62.11 & 1.24 & 12.11 & 24.53 & 24.22 & 11.97 & 25.92 & 23.64 \\
\hline 49 & SC1 & B31 & 270 & 1.71 & sandy clay loam & 69.49 & 2.82 & 15.51 & 30.72 & 20.44 & 9.71 & 20.80 & 18.50 \\
\hline 50 & SC1 & IIB32 & 390 & 1.74 & sandy clay & 69.31 & 2.73 & 14.09 & 30.19 & 22.30 & 7.39 & 23.30 & 19.49 \\
\hline 52 & SC9 & A11 & 4 & -- & clay loam & 54.20 & 0.33 & 12.94 & 26.22 & 14.71 & 22.13 & 23.67 & 20.69 \\
\hline 53 & SC9 & A12 & 53 & 1.55 & clay & 50.85 & 0.21 & 10.55 & 23.99 & 16.10 & 19.94 & 29.21 & 26.54 \\
\hline 54 & SC9 & IIB2 & 122 & 1.65 & sandy clay & 70.24 & 0.00 & 22.16 & 38.61 & 9.47 & 5.29 & 24.47 & 22.79 \\
\hline 55 & SC9 & IIIC1 & 191 & 1.22 & sandy clay loam & 60.11 & 0.22 & 8.35 & 22.75 & 28.79 & 26.22 & 13.67 & 9.89 \\
\hline 56 & SC9 & IVC2 & 280 & 1.66 & clay & 54.03 & 9.13 & 12.54 & 12.83 & 19.53 & 16.43 & 29.54 & 24.68 \\
\hline 57 & SC9 & VC3 & 420 & 1.64 & sandy clay loam & 75.84 & 2.93 & 27.41 & 26.76 & 18.74 & 11.38 & 12.78 & 8.99 \\
\hline 59 & SC7 & A12 & 46 & -- & sandy clay loam & 54.86 & 1.96 & 9.40 & 19.63 & 23.87 & 29.35 & 15.79 & 13.12 \\
\hline 61 & SC7 & B12 & 93 & -- & clay loam & 52.64 & 2.49 & 9.15 & 18.91 & 22.09 & 27.02 & 20.34 & 18.01 \\
\hline 62 & SC7 & B13 & 107 & -- & clay loam & 51.10 & 2.09 & 8.69 & 18.74 & 21.57 & 24.90 & 24.00 & 23.25 \\
\hline \multicolumn{14}{|c|}{ Alluvium of quarry terrace } \\
\hline 65 & SC6 & A11 & 2 & -- & clay & 0.00 & 0.00 & 0.00 & 0.00 & 0.00 & 0.00 & 19.06 & 14.80 \\
\hline 66 & SC6 & A12 & 16 & 1.53 & clay loam & 51.55 & 3.27 & 10.75 & 12.86 & 24.67 & 27.12 & 21.33 & 17.60 \\
\hline 67 & SC6 & A2 & 24 & 1.49 & sandy clay loam & 47.79 & 3.17 & 10.13 & 12.08 & 22.41 & 27.62 & 24.59 & -- \\
\hline 68 & SC6 & B21 & 43 & 1.48 & clay & 45.63 & 2.69 & 9.31 & 11.38 & 22.25 & 27.23 & 27.14 & 25.52 \\
\hline 69 & SC6 & IIB22 & 74 & 1.61 & clay & 38.39 & 2.44 & 8.47 & 10.44 & 17.05 & 12.89 & 48.72 & 45.35 \\
\hline 70 & SC6 & IIB23 & 96 & 1.63 & clay & 37.64 & 2.41 & 8.49 & 10.33 & 16.41 & 5.89 & 56.47 & 52.00 \\
\hline 71 & SC6 & IIIB31 & 185 & 1.68 & sandy clay & 60.48 & 5.29 & 13.50 & 14.69 & 27.00 & 13.53 & 26.00 & 19.78 \\
\hline 72 & SC6 & IIIB32 & 264 & 1.56 & sandy clay loam & 63.28 & 0.97 & 4.86 & 8.96 & 48.49 & 17.11 & 19.61 & 13.87 \\
\hline 73 & SC6 & IIIB33 & 314 & 1.63 & sandy clay loam & 77.53 & 0.77 & 14.87 & 23.13 & 38.77 & 10.75 & 11.72 & 9.21 \\
\hline
\end{tabular}




\begin{tabular}{|c|c|c|c|c|c|c|c|c|c|c|c|c|c|}
\hline No. & Sample & Horizon & $\begin{array}{c}\text { Basal } \\
\text { Depth } \\
(\mathrm{cm})\end{array}$ & $\begin{array}{c}\text { Bulk } \\
\text { Density } \\
\left(\mathrm{g} / \mathrm{cm}^{3}\right)\end{array}$ & Texture & $\begin{array}{c}\text { Total } \\
\text { sand } \\
(\%)\end{array}$ & $\begin{array}{c}\text { Very } \\
\text { coarse } \\
\text { sand } \\
(\%)\end{array}$ & $\begin{array}{c}\text { Coarse } \\
\text { sand } \\
(\%)\end{array}$ & $\begin{array}{c}\text { Medium } \\
\text { sand } \\
(\%)\end{array}$ & $\begin{array}{c}\text { Fine, very } \\
\text { fine sand } \\
(\%)\end{array}$ & $\begin{array}{l}\text { Silt } \\
(\%)\end{array}$ & $\begin{array}{c}<2-\mathrm{mm} \\
\text { clay } \\
(\%)\end{array}$ & $\begin{array}{c}<1-\mathrm{mm} \\
\text { clay } \\
(\%)\end{array}$ \\
\hline 74 & SC6 & IVC10x & 474 & 1.55 & sand & 93.35 & 0.64 & 25.51 & 39.65 & 27.54 & 4.38 & 2.27 & 1.97 \\
\hline 75 & SC8 & A11 & 9 & -- & clay loam & 42.12 & 0.21 & 7.87 & 15.76 & 18.28 & 37.88 & 20.00 & 16.49 \\
\hline 76 & SC8 & A12 & 27 & 1.44 & loam & 42.47 & 0.54 & 8.71 & 15.16 & 18.06 & 33.86 & 23.67 & 0.00 \\
\hline 77 & SC8 & A2 & 50 & 1.68 & clay loam & 41.97 & 0.43 & 7.92 & 14.86 & 18.98 & 32.65 & 25.38 & 21.26 \\
\hline 78 & SC8 & IIB2t & 110 & 1.64 & clay & 34.87 & 0.10 & 7.43 & 14.03 & 13.30 & 13.90 & 51.23 & 49.21 \\
\hline 79 & SC8 & IIIB31 & 191 & 1.66 & sandy clay & 69.95 & 0.00 & 9.44 & 38.78 & 21.73 & 6.94 & 23.11 & 20.81 \\
\hline 80 & SC8 & IIIB32 & 259 & 1.72 & sandy clay & 61.61 & 0.10 & 2.95 & 17.72 & 40.84 & 16.03 & 22.36 & 19.65 \\
\hline 81 & SC8 & IVC1 & 328 & 1.94 & clay & 41.34 & 0.30 & 5.47 & 15.40 & 20.16 & 27.29 & 31.37 & 27.25 \\
\hline 82 & SC8 & VC2 & 360 & 1.77 & clay & 55.20 & 0.82 & 7.45 & 23.47 & 23.47 & 13.86 & 30.94 & 27.76 \\
\hline 83 & SC8 & VIC3 & 413 & 1.74 & sandy clay & 64.22 & 1.07 & 10.12 & 24.28 & 28.75 & 10.86 & 24.92 & 23.43 \\
\hline 84 & SC8 & VIC4 & 480 & 1.61 & sand & 88.66 & 5.77 & 42.61 & 31.48 & 8.80 & 5.39 & 5.95 & -- \\
\hline
\end{tabular}


Extractive chemical analyses

All analyses in $\mathrm{mg} / 100 \mathrm{~g}$ soil

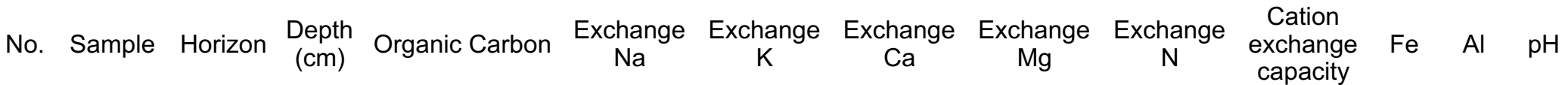

\begin{tabular}{|c|c|c|c|c|c|c|c|c|c|c|c|c|c|}
\hline \multicolumn{14}{|c|}{ Alluvium of Davenport terrace } \\
\hline 1 & SC2 & A1 & 29 & 1.07 & 0.24 & 0.13 & 6.62 & 0.89 & -- & 5.29 & 0.48 & 0.09 & 6.81 \\
\hline 2 & $\mathrm{SC} 2$ & A2 & 50 & 0.29 & 0.29 & 0.07 & 3.56 & 0.53 & -- & 5.38 & 0.36 & 0.05 & 7.22 \\
\hline 3 & $\mathrm{SC} 2$ & B1 & 90 & 0.12 & 0.29 & 0.07 & 4.61 & 1.25 & -- & 7.20 & 0.50 & 0.06 & 7.25 \\
\hline 4 & $\mathrm{SC} 2$ & B2 & 106 & 0.18 & 0.56 & 0.10 & 5.16 & 3.14 & -- & 9.84 & 0.54 & 0.07 & 7.41 \\
\hline 5 & $\mathrm{SC} 2$ & B3 & 170 & 0.03 & 0.84 & 0.10 & 3.78 & 8.05 & -- & 12.85 & 0.72 & 0.09 & 7.42 \\
\hline 6 & $\mathrm{SC} 2$ & C10x & 200 & 0.07 & 0.29 & 0.07 & 1.47 & 3.14 & -- & 5.01 & 0.60 & 0.05 & 7.34 \\
\hline 7 & $\mathrm{SC} 2$ & IIC2 & 457 & 0.08 & 0.40 & 0.09 & 3.01 & 2.68 & -- & 6.38 & 0.49 & 0.04 & 7.14 \\
\hline 8 & $\mathrm{SC} 2$ & $\mathrm{IICn}$ & 518 & 0.07 & 0.34 & 0.07 & 1.80 & 3.86 & -- & 6.11 & 0.51 & 0.05 & 7.52 \\
\hline \multicolumn{14}{|c|}{ Alluvium of Wilder terrace } \\
\hline 9 & SC3 & A11 & 8 & 2.36 & 0.14 & 0.75 & 10.43 & 3.86 & -- & 17.78 & 1.50 & 0.17 & 6.64 \\
\hline 10 & SC3 & A12 & 23 & 1.84 & 0.04 & 0.35 & 10.54 & 1.25 & -- & 17.69 & 1.62 & 0.20 & 6.48 \\
\hline 11 & SC3 & A3 & 87 & 0.81 & 0.14 & 0.13 & 7.86 & 1.07 & -- & 13.31 & 1.73 & 0.26 & 6.87 \\
\hline 12 & SC3 & IIB21t & 126 & 0.44 & 0.24 & 0.10 & 6.96 & 0.62 & -- & 12.13 & 1.76 & 0.29 & 7.04 \\
\hline 13 & SC3 & IIB22t & 190 & 0.19 & 0.14 & 0.09 & 3.66 & 0.98 & -- & 9.57 & 1.89 & 0.28 & 7.05 \\
\hline 14 & SC3 & IIIB23t & 240 & 0.18 & 0.14 & 0.09 & 3.06 & 2.96 & -- & 10.21 & 0.16 & 0.26 & 6.50 \\
\hline 15 & SC3 & IIIB31 & 325 & 0.17 & 0.24 & 0.09 & 2.73 & 3.22 & -- & 8.84 & 1.77 & 0.24 & 6.88 \\
\hline 16 & SC3 & IIIB32 & 390 & 0.17 & 0.19 & 0.09 & 2.45 & 2.86 & -- & 7.38 & 1.43 & 0.20 & 6.68 \\
\hline 28 & SC5 & All & 2.5 & 4.12 & 0.24 & 0.86 & 9.21 & 1.47 & -- & 16.59 & 1.04 & 0.17 & 6.10 \\
\hline 29 & SC5 & A12 & 25 & 1.63 & 0.00 & 0.28 & 8.23 & 0.66 & -- & 13.31 & 1.41 & 0.24 & 6.37 \\
\hline 30 & SC5 & B1 & 60 & 0.77 & 0.08 & 0.20 & 6.43 & 0.44 & -- & 11.49 & 1.95 & 0.38 & 6.45 \\
\hline 31 & SC5 & B21t & 85 & 0.45 & 0.30 & 0.14 & 6.35 & 1.03 & -- & 12.22 & 2.34 & 0.48 & 6.53 \\
\hline 32 & SC5 & B22t & 111 & 0.40 & 0.30 & 0.12 & 6.05 & 2.86 & -- & 14.77 & 2.82 & 0.45 & 6.56 \\
\hline 33 & SC5 & B31t & 158 & 0.27 & 0.24 & 0.07 & 2.27 & 2.64 & -- & 9.94 & 2.41 & 0.38 & 5.44 \\
\hline 34 & SC5 & B32 & 220 & 0.25 & 0.19 & 0.07 & 1.37 & 2.35 & -- & 8.39 & 2.09 & 0.27 & 5.27 \\
\hline 35 & SC5 & B33 & 270 & 0.20 & 0.24 & 0.09 & 1.29 & 2.49 & -- & 7.75 & 1.77 & 0.27 & 5.21 \\
\hline 36 & SC5 & B34 & 325 & 0.19 & 0.00 & 0.10 & 1.82 & 3.60 & -- & 9.75 & 2.05 & 0.27 & 5.15 \\
\hline 37 & SC5 & B35 & 415 & 0.18 & 0.24 & 0.14 & 2.65 & 4.47 & -- & 11.12 & 1.70 & 0.24 & 5.49 \\
\hline 38 & SC5 & Cox & 480 & 0.10 & 0.30 & 0.13 & 2.20 & 3.45 & -- & 7.93 & 0.75 & 0.11 & 5.62 \\
\hline 39 & SC5core & Cox & 640 & -- & 0.19 & 0.20 & 3.64 & 3.96 & -- & 8.93 & 0.79 & 0.12 & 6.26 \\
\hline
\end{tabular}


No. Sample Horizon Depth Organic Carbon Exchange Exchange Exchange Exchange Exchange Cation

\begin{tabular}{|c|c|c|c|c|c|c|c|c|c|c|c|c|c|}
\hline \multicolumn{14}{|c|}{ Alluvium of Davenport terrace } \\
\hline 40 & SC5core & Cox & 762 & -- & 0.35 & 0.25 & 5.14 & 6.38 & -- & 13.95 & 0.91 & 0.12 & 6.19 \\
\hline 41 & SC5core & Cox & 823 & -- & 0.19 & 0.14 & 2.27 & 2.06 & -- & 5.01 & 0.30 & 0.08 & 6.20 \\
\hline 42 & SC5core & Cox & 1128 & 0.17 & 0.19 & 0.20 & 10.41 & 1.10 & -- & 12.40 & 1.57 & 0.08 & 7.55 \\
\hline \multicolumn{14}{|c|}{ Alluvium of Black Rock terrace } \\
\hline 43 & SC1 & A11 & 3 & 4.86 & 0.56 & 0.63 & 6.57 & 1.70 & -- & 18.14 & 1.27 & 0.24 & 5.53 \\
\hline 44 & SC1 & A12 & 50 & 0.75 & 0.19 & 0.13 & 2.24 & 0.98 & -- & 9.94 & 1.38 & 0.29 & 5.71 \\
\hline 45 & SC1 & B11 & 65 & 0.54 & 0.19 & 0.12 & 1.96 & 1.25 & -- & 7.93 & 1.62 & 0.32 & 6.00 \\
\hline 46 & SC1 & B12 & 84 & 0.49 & 0.19 & 0.10 & 2.07 & 1.87 & -- & 8.44 & 1.53 & 0.20 & 5.90 \\
\hline 47 & SC1 & B21t & 128 & 0.25 & 0.40 & 0.12 & 4.39 & 5.31 & -- & 14.13 & 3.24 & 0.47 & 6.00 \\
\hline 48 & SC1 & B22t & 195 & 0.22 & 0.40 & 0.10 & 2.89 & 3.14 & -- & 10.21 & 4.18 & 0.45 & 6.03 \\
\hline 49 & SC1 & B31 & 270 & 0.16 & 0.45 & 0.12 & 2.95 & 2.96 & -- & 9.39 & 2.09 & 0.35 & 6.34 \\
\hline 50 & SC1 & IIB32 & 390 & 0.16 & 0.50 & 0.13 & 3.06 & 3.04 & -- & 10.21 & 2.00 & 0.12 & 6.35 \\
\hline 52 & SC9 & A11 & 4 & 5.42 & 0.82 & 1.17 & 13.84 & 1.54 & -- & 23.25 & 1.64 & 0.26 & 5.59 \\
\hline 53 & SC9 & A12 & 53 & 2.32 & 0.64 & 0.48 & 12.13 & 0.69 & -- & 17.32 & 2.14 & 0.35 & 6.09 \\
\hline 54 & SC9 & IIB2 & 122 & 0.12 & 0.82 & 0.10 & 1.17 & 1.15 & -- & 7.93 & 2.31 & 0.30 & 4.43 \\
\hline 55 & SC9 & IIIC1 & 191 & 0.05 & 0.70 & 0.10 & 0.81 & 1.39 & -- & 4.56 & 0.89 & 0.12 & 4.57 \\
\hline 56 & SC9 & IVC2 & 280 & 0.04 & 1.18 & 0.10 & 2.71 & 5.29 & -- & 14.31 & 2.12 & 0.15 & 4.48 \\
\hline 57 & SC9 & VC3 & 420 & 0.05 & 0.76 & 0.08 & 1.27 & 2.24 & -- & 5.92 & 0.59 & 0.08 & 4.83 \\
\hline 59 & SC7 & A12 & 46 & 1.82 & 0.53 & 0.34 & 3.27 & 0.38 & -- & 10.30 & 1.36 & 0.26 & 5.68 \\
\hline 60 & SC7 & B11 & 76 & 0.76 & 0.59 & 0.20 & 1.94 & 0.61 & -- & 7.75 & 1.54 & 0.32 & 5.65 \\
\hline 61 & SC7 & B12 & 93 & 0.41 & 0.53 & 0.15 & 2.24 & 1.00 & -- & 7.11 & 1.74 & 0.35 & 5.90 \\
\hline 62 & $\mathrm{SC} 7$ & B13 & 107 & 4.00 & 0.59 & 0.15 & 2.55 & 1.54 & -- & 8.11 & 2.15 & 0.44 & 5.99 \\
\hline \multicolumn{14}{|c|}{ Alluvium of quarry terrace } \\
\hline 65 & SC6 & A11 & 2 & 2.32 & 0.24 & 0.62 & 6.20 & 1.54 & $\overline{--}$ & 17.60 & 1.31 & 0.23 & 5.50 \\
\hline 66 & SC6 & A12 & 16 & 1.54 & 0.19 & 0.23 & 3.26 & 0.95 & -- & 12.03 & 1.81 & 0.31 & 5.26 \\
\hline 67 & SC6 & $\mathrm{A} 2$ & 24 & 1.37 & 0.08 & 0.25 & 2.43 & 1.03 & -- & 11.94 & 2.07 & 0.37 & 5.09 \\
\hline 68 & SC6 & B21 & 43 & 0.62 & 0.14 & 0.22 & 2.20 & 0.88 & -- & 11.12 & 2.17 & 0.40 & 5.08 \\
\hline 69 & SC6 & IIB22 & 74 & 0.29 & 0.41 & 0.23 & 1.82 & 2.72 & -- & 14.95 & 3.73 & 0.61 & 5.10 \\
\hline 70 & SC6 & IIB23 & 96 & 0.47 & 0.30 & 0.14 & 1.21 & 2.42 & -- & 15.77 & 4.34 & 0.62 & 4.89 \\
\hline 71 & SC6 & IIIB31 & 185 & 0.28 & 0.30 & 0.10 & 0.53 & 1.98 & -- & 10.67 & 1.73 & 0.29 & 4.84 \\
\hline 72 & SC6 & IIIB32 & 264 & 0.31 & 0.24 & 0.10 & 1.06 & 2.93 & -- & 13.13 & 2.12 & 0.31 & 4.83 \\
\hline 73 & SC6 & IIIB33 & 314 & 0.25 & 0.41 & 0.10 & 1.29 & 2.93 & -- & 10.76 & 1.56 & 0.23 & 4.97 \\
\hline 74 & SC6 & IVC1ox & 474 & 0.25 & 0.64 & 0.23 & 1.43 & 2.01 & -- & 6.11 & 0.33 & 0.12 & 5.49 \\
\hline
\end{tabular}


Table 4

$\begin{array}{cccccccccccc}\text { No. Sample Horizon } & \begin{array}{c}\text { Depth } \\ (\mathrm{cm})\end{array}\end{array}$


Mineralogy

Analysts: Jacob Aniku, University of California, Davis

+++,dominant; ++, moderate; +,trace; 0, not detected; --,not analyzed; $X X$,dominant; $X$,present.

\begin{tabular}{|c|c|c|c|c|c|c|c|c|c|}
\hline Sample & Horizon & $\begin{array}{c}\text { Depth } \\
\text { (cm) }\end{array}$ & Kaolinite & Mica & $\begin{array}{c}\text { Interstratified } \\
\text { Chlorite- } \\
\text { Vermuculite }\end{array}$ & Gibbsite & $\begin{array}{l}\text { Expanding } \\
\text { Clays }\end{array}$ & Quartz & Feldspar \\
\hline \multicolumn{10}{|c|}{ Alluvium of Davenport terrace } \\
\hline \multirow[t]{3}{*}{ SC2 } & $E$ & 50 & +++ & + & ++ & + & + & $\mathrm{XX}$ & $\mathrm{X}$ \\
\hline & $\mathrm{Bt} 1$ & 106 & +++ & + & ++ & + & + & $X X$ & $X$ \\
\hline & $\mathrm{C} 1$ & 200 & + & + & + & + & + & $\mathrm{X}$ & $\mathrm{X}$ \\
\hline \multicolumn{10}{|c|}{ Alluvium of lower Wilder terrace } \\
\hline \multirow[t]{3}{*}{ SC5 } & $\mathrm{A} 2$ & 25 & +++ & + & ++ & + & 0 & $\mathrm{XX}$ & $\mathrm{X}$ \\
\hline & Bt2 & 111 & +++ & + & ++ & + & + & $X X$ & $X$ \\
\hline & $\mathrm{BC} 1$ & 220 & +++ & + & + & + & + & $X X$ & $\mathrm{X}$ \\
\hline \multicolumn{10}{|c|}{ Alluvium of Black Rock terrace } \\
\hline \multirow[t]{3}{*}{ SC1 } & $\mathrm{A} 2$ & 50 & +++ & + & ++ & + & 0 & $\mathrm{XX}$ & $\mathrm{X}$ \\
\hline & $\mathrm{Bt} 2$ & 195 & +++ & + & ++ & + & + & $X X$ & $X$ \\
\hline & $2 \mathrm{CB}$ & 390 & + & 0 & + & 0 & 0 & $X X$ & $\mathrm{X}$ \\
\hline \multicolumn{10}{|c|}{ Alluvium of quarry terrace } \\
\hline \multirow[t]{4}{*}{ SC6 } & $\mathrm{A} 2$ & 16 & +++ & + & ++ & + & + & $\mathrm{XX}$ & $\mathrm{X}$ \\
\hline & $2 \mathrm{Bt} 2$ & 74 & +++ & + & ++ & + & 0 & $X X$ & $X$ \\
\hline & $2 \mathrm{Bt} 3$ & 96 & +++ & + & ++ & + & + & $X X$ & $X$ \\
\hline & $3 \mathrm{CB}$ & 314 & +++ & + & + & + & 0 & $X X$ & $x$ \\
\hline
\end{tabular}


Total chemical analyses of the fine $(<47 \mu \mathrm{m})$ fraction by $\mathrm{X}$-ray fluorescence

Analysts: A.J. Bartel, K.Stewart, and J. Taggart and R. Johnson and K. Dennen under J.R. Lindsay

All analyses in weight percent

Basal

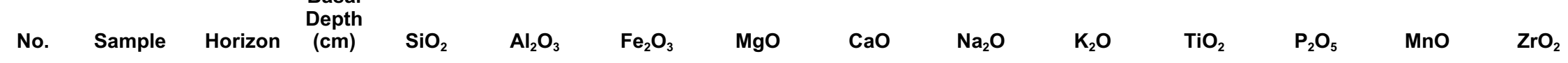

\begin{tabular}{|c|c|c|c|c|c|c|c|c|c|c|c|c|c|c|}
\hline \multicolumn{15}{|c|}{ Alluvium of Davenport terrace } \\
\hline 1 & SC2 & A1 & 29 & 71.00 & 13.70 & 3.15 & 0.51 & 1.33 & 2.32 & 1.69 & 0.76 & 0.12 & 0.05 & 0.04 \\
\hline 4 & SC2 & B2 & 106 & 61.60 & 17.40 & 5.94 & 0.86 & 1.28 & 1.92 & 1.26 & 0.85 & 0.05 & 0.03 & 0.03 \\
\hline 8 & SC2 & $\mathrm{IICn}$ & 518 & 50.60 & 16.00 & 12.40 & 3.54 & 2.54 & 1.82 & 1.28 & 0.80 & 0.17 & 0.13 & 0.02 \\
\hline \multicolumn{15}{|c|}{ Alluvium of Wilder terrace } \\
\hline 29 & SC5 & A12 & 25 & 92.50 & 3.55 & 0.76 & 0.10 & 0.25 & 0.51 & 1.58 & 0.31 & 0.05 & 0.02 & 0.02 \\
\hline 32 & SC5 & B22t & 111 & 47.30 & 25.00 & 8.77 & 0.60 & 0.28 & 0.26 & 0.79 & 0.88 & 0.11 & 0.02 & 0.07 \\
\hline 38 & SC5 & Cox & 480 & 49.90 & 26.30 & 6.67 & 0.91 & 0.39 & 1.05 & 1.21 & 0.62 & 0.07 & 0.02 & 0.02 \\
\hline \multicolumn{15}{|c|}{ Alluvium of Black Rock terrace } \\
\hline 44 & SC1 & A12 & 50 & 80.80 & 8.15 & 2.94 & 0.23 & 0.24 & 0.45 & 0.83 & 0.69 & 0.06 & 0.02 & 0.03 \\
\hline 48 & SC1 & B22t & 195 & 48.20 & 22.40 & 12.90 & 0.64 & 0.25 & 0.61 & 0.97 & 0.97 & 0.06 & 0.02 & 0.02 \\
\hline 50 & SC1 & IIB32 & 390 & 86.60 & 5.81 & 2.09 & 0.24 & 0.51 & 1.17 & 1.88 & 0.76 & 0.05 & 0.03 & 0.06 \\
\hline \multicolumn{15}{|c|}{ Alluvium of quarry terrace } \\
\hline 65 & SC6 & A11 & 2 & 69.30 & 12.40 & 4.11 & 0.32 & 0.36 & 0.79 & 1.96 & 1.03 & 0.13 & 0.02 & 0.05 \\
\hline 66 & SC6 & A12 & 16 & 64.40 & 16.70 & 5.62 & 0.50 & 0.30 & 0.73 & 1.77 & 1.09 & 0.14 & 0.03 & 0.05 \\
\hline 67 & SC6 & $\mathrm{A} 2$ & 24 & 61.30 & 18.80 & 6.53 & 0.52 & 0.25 & 0.66 & 1.57 & 1.08 & 0.14 & 0.03 & 0.05 \\
\hline 68 & SC6 & B21 & 43 & 60.00 & 19.10 & 6.56 & 0.52 & 0.25 & 0.69 & 1.52 & 1.05 & 0.14 & 0.03 & 0.04 \\
\hline 69 & SC6 & IIB22 & 74 & 46.70 & 26.10 & 9.42 & 0.57 & 0.09 & 0.29 & 0.77 & 0.96 & 0.09 & 0.02 & 0.03 \\
\hline 70 & SC6 & IIB23 & 96 & 44.70 & 26.70 & 10.10 & 0.60 & 0.07 & 0.26 & 0.64 & 0.97 & 0.09 & 0.02 & 0.03 \\
\hline 71 & SC6 & IIIB31 & 185 & 49.80 & 24.60 & 8.38 & 0.77 & 0.05 & 0.34 & 1.39 & 0.98 & 0.07 & 0.02 & 0.03 \\
\hline 72 & SC6 & IIIB32 & 264 & 49.10 & 23.60 & 10.20 & 0.99 & 0.12 & 0.63 & 0.98 & 0.99 & 0.07 & 0.02 & 0.02 \\
\hline 73 & SC6 & IIIB33 & 314 & 47.00 & 26.00 & 8.57 & 0.86 & 0.18 & 0.74 & 1.17 & 0.74 & 0.09 & 0.02 & 0.02 \\
\hline 74 & SC6 & IVC10x & 474 & 47.80 & 29.30 & 4.17 & 0.64 & 0.51 & 1.14 & 1.54 & 0.50 & 0.05 & 0.02 & 0.02 \\
\hline 76 & SC8 & A12 & 27 & 67.70 & 15.10 & 5.01 & 0.38 & 0.33 & 0.82 & 1.67 & 0.99 & 0.10 & 0.02 & 0.05 \\
\hline 80 & SC8 & IIIB32 & 259 & 58.10 & 19.20 & 7.15 & 0.63 & 0.37 & 1.28 & 1.02 & 0.74 & 0.05 & 0.02 & 0.03 \\
\hline 84 & SC8 & VIC4 & 480 & 54.00 & 24.20 & 4.46 & 0.89 & 0.93 & 1.65 & 1.16 & 0.71 & 0.05 & 0.02 & 0.04 \\
\hline
\end{tabular}


Total chemical analyses of $<2 \mathrm{~mm}$ fraction by X-ray fluorescence

Analysts: A.J. Bartel, K.Stewart, and J. Taggart and R. Johnson and K. Dennen under J.R. Lindsay

All analyses in weight percent

No. Basal Depth

\begin{tabular}{|c|c|c|c|c|c|c|c|c|c|c|c|c|c|c|}
\hline \multicolumn{15}{|c|}{ Alluvium of Davenport terrace } \\
\hline 2 & SC2 & A2 & 50 & 79.80 & 9.69 & 1.83 & 0.41 & 1.27 & 1.93 & 1.78 & 0.52 & 0.05 & 0.03 & 0.03 \\
\hline 4 & $\mathrm{SC} 2$ & B2 & 106 & 66.90 & 14.80 & 4.65 & 0.80 & 1.65 & 2.24 & 1.46 & 0.86 & 0.05 & 0.04 & 0.05 \\
\hline \multicolumn{15}{|c|}{ Alluvium of Wilder terrace } \\
\hline 29 & SC5 & A12 & 25 & 69.50 & 12.10 & 3.84 & 0.38 & 0.64 & 0.68 & 1.85 & 0.81 & 0.17 & 0.09 & 0.04 \\
\hline 32 & SC5 & $\mathrm{B} 22 \mathrm{t}$ & 111 & 59.00 & 19.40 & 6.83 & 0.44 & 0.25 & 0.20 & 0.97 & 0.75 & 0.09 & 0.02 & 0.02 \\
\hline 38 & SC5 & Cox & 480 & 63.40 & 18.40 & 4.03 & 0.50 & 0.36 & 1.07 & 1.92 & 0.40 & 0.05 & 0.04 & 0.01 \\
\hline \multicolumn{15}{|c|}{ Alluvium of Black Rock terrace } \\
\hline 44 & SC1 & A12 & 50 & 72.30 & 11.90 & 4.08 & 0.31 & 0.34 & 0.70 & 1.67 & 0.96 & 0.08 & 0.02 & 0.07 \\
\hline 48 & SC1 & $\mathrm{B} 22 \mathrm{t}$ & 195 & 63.40 & 16.00 & 6.31 & 0.45 & 0.26 & 0.63 & 1.16 & 0.94 & 0.05 & 0.02 & 0.05 \\
\hline 50 & SC1 & IIB32 & 390 & 61.90 & 19.10 & 4.85 & 0.64 & 0.99 & 1.63 & 1.64 & 0.57 & 0.07 & 0.02 & 0.02 \\
\hline \multicolumn{15}{|c|}{ Alluvium of quarry terrace } \\
\hline 65 & SC6 & A11 & 2 & 67.00 & 10.50 & 3.59 & 0.37 & 0.44 & 0.51 & 1.59 & 0.88 & 0.12 & 0.04 & 0.05 \\
\hline 66 & SC6 & A12 & 16 & 68.00 & 13.20 & 4.41 & 0.37 & 0.31 & 0.56 & 1.66 & 0.95 & 0.12 & 0.04 & 0.05 \\
\hline 67 & SC6 & $\mathrm{A} 2$ & 24 & 67.20 & 14.40 & 5.03 & 0.42 & 0.25 & 0.40 & 1.56 & 1.00 & 0.12 & 0.03 & 0.05 \\
\hline 68 & SC6 & B21 & 43 & 65.90 & 15.20 & 5.19 & 0.42 & 0.24 & 0.47 & 1.51 & 0.96 & 0.11 & 0.03 & 0.05 \\
\hline 69 & SC6 & IIB22 & 74 & 54.90 & 20.80 & 8.20 & 0.42 & 0.08 & 0.17 & 0.87 & 0.87 & 0.08 & 0.02 & 0.03 \\
\hline 70 & SC6 & IIB23 & 96 & 60.70 & 17.50 & 7.77 & 0.35 & 0.05 & 0.15 & 0.68 & 0.82 & 0.06 & 0.02 & 0.03 \\
\hline 71 & SC6 & IIIB31 & 185 & 64.70 & 16.60 & 5.98 & 0.56 & 0.05 & 0.28 & 1.37 & 0.83 & 0.05 & 0.02 & 0.04 \\
\hline 72 & SC6 & IIIB32 & 264 & 59.90 & 17.90 & 7.41 & 0.78 & 0.20 & 0.72 & 1.13 & 1.12 & 0.05 & 0.02 & 0.04 \\
\hline 73 & SC6 & IIIB33 & 314 & 61.20 & 18.90 & 5.67 & 0.56 & 0.26 & 0.80 & 1.86 & 0.66 & 0.06 & 0.02 & 0.03 \\
\hline 74 & SC6 & IVC10x & 474 & 75.70 & 13.20 & 1.27 & 0.22 & 0.45 & 1.12 & 2.77 & 0.18 & 0.05 & 0.02 & 0.01 \\
\hline 76 & SC8 & A12 & 27 & 72.80 & 9.65 & 3.46 & 0.28 & 0.51 & 0.61 & 1.25 & 0.84 & 0.10 & 0.02 & 0.04 \\
\hline 80 & SC8 & IIIB32 & 259 & 67.60 & 14.30 & 6.02 & 0.33 & 0.12 & 0.34 & 0.46 & 0.57 & 0.05 & 0.02 & 0.02 \\
\hline 84 & SC8 & VIC4 & 480 & 74.10 & 14.30 & 1.33 & 0.29 & 1.49 & 2.45 & 1.81 & 0.25 & 0.05 & 0.02 & 0.10 \\
\hline
\end{tabular}

